\section{Advances in the}

\section{treatment of}

\section{melanoma}

Katie E Lacy, consultant dermatologist; Sophia N Karagiannis, NIHR senior research fellow; Frank $\mathrm{O}$ Nestle, Mary Dunhill chair of cutaneous medicine and immunotherapy

NIHR Biomedical Research Centre at Guy's and St Thomas's Hospitals and King's College London; Cutaneous Medicine and Immunotherapy, St John's Institute of Dermatology, Division of Genetics and Molecular Medicine, King's College London School of Medicine, Guy's Hospital, KCL, London

Malignant melanoma is an aggressive form of skin cancer arising from malignantly transformed melanocytes. The incidence and mortality from melanoma are currently rising, placing significant demands on healthcare provision and representing a major public health issue. Surgical excision remains the standard of care for the treatment of primary melanomas. Excision of thin tumours less than $1 \mathrm{~mm}$ in thickness can result in an over $95 \%$ five-year survival rate. ${ }^{1}$

Despite this however, $20 \%$ of primary melanomas will progress to metastatic disease for which the treatment options are limited and the prognosis poor with a median survival of 8-18 months. ${ }^{1}$ Over the last year significant progress has been made in the treatment of advanced stage disease with the reporting of large phase III studies demonstrating improved survival in patients with metastatic melanoma, followed by Food and Drug Administration (FDA) approval of new therapeutic agents (Table 1 ).

\section{Longstanding conventional therapies for melanoma}

\section{Chemotherapeutics}

The need for development of novel therapeutics is highlighted by the fact that

Table 1. Food \& Drug Administration approved agents for the treatment of melanoma.

\begin{tabular}{|c|c|c|}
\hline Year of approval & Agent & Type of agent and actions \\
\hline 2011 & Vemurafenib & BRAF V600E kinase inhibitor \\
\hline 2011 & Ipilimumab & $\begin{array}{l}\text { Humanised mAB targeting the inhibitory } \\
\text { molecule CTLA-4 }\end{array}$ \\
\hline 2011 & Pegylated IFN- $\alpha 2 \beta$ & $\begin{array}{l}\text { Modified (pegylated) IFN has actions as for } \\
\text { IFN- } \alpha 2 \beta \text {, with increased half life and enhanced } \\
\text { therapeutic efficacy }\end{array}$ \\
\hline 1998 & High dose IL-2 & $\begin{array}{l}\text { Cytokine therapy aimed at promoting T-cell } \\
\text { activation and proliferation }\end{array}$ \\
\hline 1995 & IFN- $\alpha 2 \beta$ & $\begin{array}{l}\text { Interferon enhances the antitumour immune } \\
\text { response in addition to having anti-angiogenic } \\
\text { and pro-apoptotic properties }\end{array}$ \\
\hline 1975 & Dacarbazine & $\begin{array}{l}\text { Alkylating agent inducing cell cycle arrest and } \\
\text { tumour cell apoptosis }\end{array}$ \\
\hline
\end{tabular}

$\mathrm{mAB}=$ monoclonal antibody; IFN = interferon; $\mathrm{IL}=$ interleukin.

standard chemotherapeutic regimens have little impact on survival for advanced disease. An analysis of randomised controlled trials demonstrated no superiority of chemotherapy over supportive care and, for patients who do respond, the remissions are usually of short duration. ${ }^{2}$ The alkylating agent dacarbazine (DTIC) is currently widely used as part of standard care for the treatment of unresectable stage IV disease, in spite of a response rate of only around $20 \%$ and a median response duration of 4-5 months. ${ }^{2}$

Other chemotherapeutic agents including temozolomide, cisplatin, carboplatin, vinca alkaloids and taxanes are associated with poor outcomes and significant adverse side effects.

\section{Interleukin-2}

Interleuklin (IL)-2 is thought to have antitumour effects both by inducing $\mathrm{T}$ cell activation and proliferation and by stimulating host antitumour responses. The treatment of advanced disease with IL-2 alone, however, has shown limited responses in around $15 \%$ of cases, although durable remissions have been reported in some patients. ${ }^{3}$ The use of IL-2 in high doses is associated with considerable mortality, with the development of gastrointestinal, cardiovascular, renal and neurological toxicities, including the vascular leak syndrome with accumulation of fluid within the lung and liver.

\section{Interferon}

The type 1 interferons (IFN- $\alpha$, IFN- $\alpha 2 \beta$ ) have been evaluated in numerous clinical trials and shown in some studies to be beneficial in prolonging disease-free survival when used in the adjuvant setting. Recent promise has been demonstrated with a modified form of IFN, pegylated IFN- $\alpha 2 \beta$; this has a longer half-life, enhanced therapeutic efficacy and improved tolerability. A phase III study investigating the use of this agent versus observation alone as adjuvant therapy for resected stage III disease (metastatic disease within the draining lymph node basin) showed a significant improvement in relapse-free survival. ${ }^{4}$

\section{New agents for the treatment of melanoma}

It is well recognised that melanoma is an immunogenic tumour with the demonstration of $\mathrm{T}$ lymphocyte infiltrates within the tumour that correlate with improved patient survival. Despite the presence of demonstrable antitumour immune responses, tumours are able to evade the immune response and progress, a process known as immune escape. Multiple immunotherapeutic strategies have been adopted with a view 
to overcoming the immunosuppressive tumour environment and boosting antimelanoma immunity. Overall response rates have been poor in previous studies investigating immunotherapeutic modalities despite showing some tantalising results - for example, a limited number of patients developed durable responses with $\mathrm{T}$ - and dendritic cell autologous cell therapies.

\section{Ipilimumab}

Interest in immunotherapeutic approaches has been reinvigorated over the last year following promising results with ipilimumab, a human monoclonal antibody (mAB) targeting CTLA-4. CTLA-4 is expressed on the cell surface of activated $\mathrm{T}$ lymphocytes, acting as an inhibitory molecule by competing for binding to B7 with CD28, a co-stimulatory molecule also expressed on $\mathrm{T}$ lymphocytes (Fig 1).

In a recent phase III trial in patients with stage III or IV disease, ipilimumab therapy improved the median overall survival (10.1 months) compared with a peptide vaccine (gp100) (6.4 months). ${ }^{5} \mathrm{~A}$ subsequent phase III trial compared treatment with ipilimumab and DTIC versus DTIC alone, with improved survival in the group treated with ipilimumab (11.2 months vs 9.1 months). ${ }^{6}$ Importantly, although there was relatively modest improvement in overall survival, durable responses were seen in a number of patients.

Studies are now also underway to evaluate the use of ipilimumab in combinations with other therapies such as IL-2. The utility of the drug in the adjuvant setting is also being assessed.

Side effects. Side effects of ipilimumab include development of immune-related adverse effects including colitis and dermatitis that are commonly responsive to systemic steroids. However, improved clinical responses are seen in patients who develop autoimmune complications, highlighting the fine balance that needs to be achieved between stimulating antitumour responses and the development of unwanted autoimmunity with subsequent toxicities. a

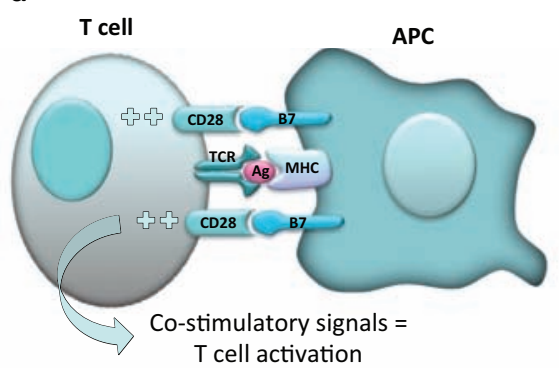

b

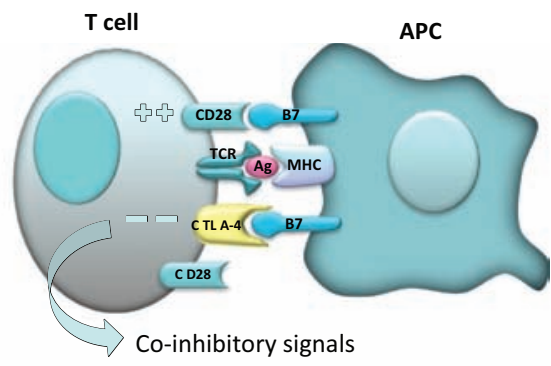

d
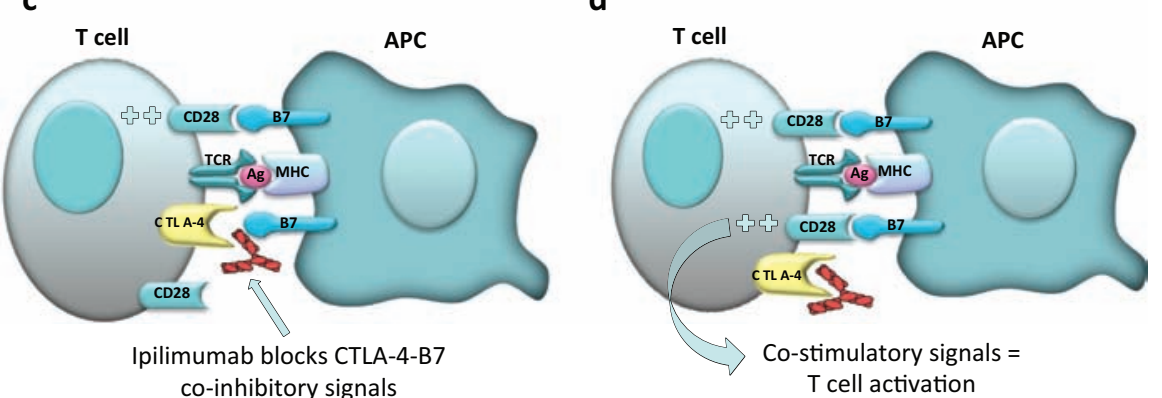

Fig 1. Mechanism of action of ipilimumab. Melanoma antigens (Ag) bound to the major histocompatibility complex (MHC) on the surface of antigen-presenting cells (APC) are presented to T-cells through the T-cell receptor (TCR): (a) T-cell activation is achieved by costimulatory signals to T-cells by CD28 receptor recognition of B7 ligands on APCs; (b) an important immunoregulatory signal is that of the cytotoxic T lymphocyte antigen-4 (CTLA-4) on T-cells which competes with CD28 for binding to B7 molecules, blocking the necessary co-stimulatory signals by APCs; (c) ipilimumab blocks binding of CTLA-4 to B7 on the surface of APCs; (d) this allows CD28-B7 complex assembly and co-stimulation, restoring T-cell activation.

\section{Mutation targeted therapies for melanoma: agents targeting BRAF mutations}

Newly emerging therapeutics that target oncogenes thought to be critical in the pathogenesis of melanoma are currently under evaluation. Around $60 \%$ of melanoma tumours are thought to have mutations in the BRAF gene, the commonest of which is V600E. BRAF gene mutations result in activation of

\section{Key points}

Melanoma incidence and mortality are increasing

Melanoma is relatively resistant to therapy with standard chemotherapeutic regimens

The small molecule inhibitor vemurafenib that targets melanomas with a common and specific mutation (BRAF V600E) has shown promise in the treatment of melanoma, representing a successful form of truly personalised therapy based on molecular testing of individual tumours

Success with the anti-CTLA-4 monoclonal antibody ipilimumab highlights the therapeutic potential of targeting antitumour immune responses

Numerous other agents are currently under development and may provide alternative therapeutic options in the future

Combinations of treatments that target different pathways involved in melanoma progression require further evaluation

KEY WORDS: immunotherapy, melanoma, small molecule inhibitor, tumour mutation 
downstream signalling through the mitogen-activated protein kinase (MAPK) pathway (Fig 2). Initial trials using the non-selective BRAF kinase inhibitor sorafenib were disappointing, but data from studies evaluating the effects of vemurafenib, a potent selective inhibitor of mutated BRAF, have shown exciting results with clinical responses in patients with BRAF V600E mutations. A recently published large multicentre phase III trial showed improved overall survival compared with patients treated with DTIC, leading to FDA approval. ${ }^{7}$ Overall survival at six months was $84 \%$ versus $64 \%$ in the vemurafenib and DTIC treated groups, respectively, with response rates of $48 \%$ versus $5 \%$. $^{7}$

Studies are also ongoing to assess the efficacy of another BRAF inhibitor, dabrafenib (GSK2118436), thought to target tumours with both BRAF V600E and BRAF V600K mutations. Early studies have shown this agent to be effective for the treatment of brain metastases $^{8}$ and a large Phase III study is currently underway.
Side effects. The most common side effects with BRAF inhibitors include the development of cutaneous squamous cell carcinomas in $18-24 \%$ of patients, photosensitivity, rash, arthralgia, fatigue, alopecia and pruritus. Unfortunately, it is now well recognised that after initial impressive responses, tumours often develop drug resistance with reactivation of the MAPK pathway. Attempts to overcome drug resistance are now underway with the introduction of MEK (mitogen activated protein kinase) inhibitors. MEK is a downstream target of BRAF in the MAPK pathway. Preliminary studies with the combined use of dabrafenib and the MEK inhibitor GSK1120212 have shown promise, with delayed emergence of resistance to dabrafenib and decreased incidence of squamous cell carcinoma development. ${ }^{9}$

\section{New therapeutic agents currently in development}

\section{Inhibitors targeting CKIT mutations}

Mutations in cKit, the receptor tyrosine kinase for stem cell factor, although

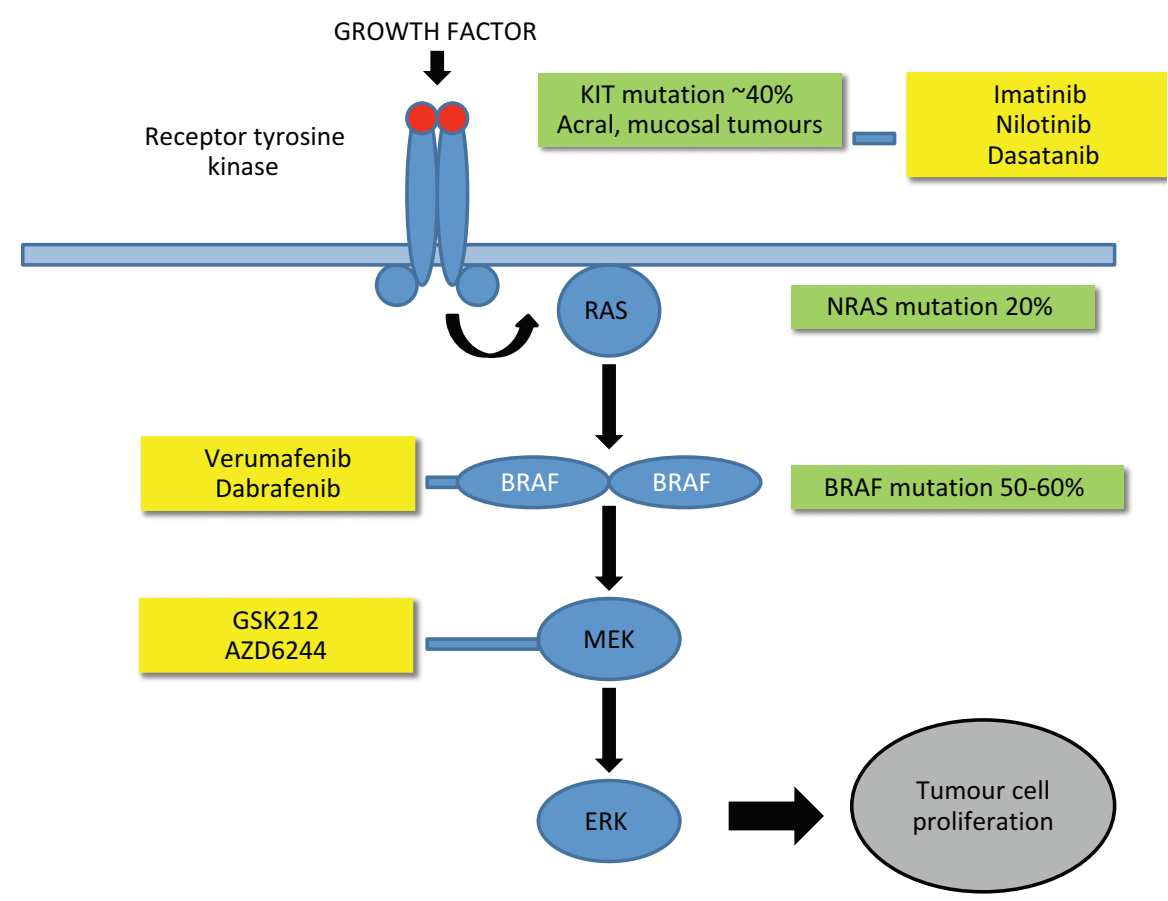

Fig 2. The mitogen-activated protein kinase (MAPK) pathway and potential therapeutic targets in melanoma. Activation of the receptor tyrosine kinase receptor by growth factors results in activation of the MAPK pathway and subsequent tumour cell proliferation. A significant proportion of melanomas contain activating mutations (green boxes) that result in activation of protein kinases (blue boxes). Various agents have been developed that target activated protein kinases with specific mutations (yellow boxes). uncommon in cutaneous melanomas, have been identified in up to $20 \%$ of acral and mucosal melanomas. ${ }^{10}$ Accumulating evidence suggests that certain small molecule inhibitors of c-KIT, including imatinib, nilotinib and dasatinib, may be of benefit in patients with c-KIT mutations. These are currently under evaluation in clinical trials. ${ }^{11-12}$

\section{Antibodies targeting vascular endothelial growth factor}

It is well recognised that the formation of new blood vessels within tumours can help to promote metastasis and tumour growth. The humanised mAB bevacizumab targets vascular endothelial growth factor (VEGF) by blocking its binding to the VEGF receptor, and is thought to inhibit angiogenesis. Bevacizumab is FDA approved for the treatment of metastatic colorectal cancer, breast cancer and non-small cell lung cancer in combination with chemotherapy. Its use in the treatment of melanoma is currently under evaluation. Phase II studies have shown some activity against melanoma in combination with IFN and fotmustine. ${ }^{13}$ A large Phase III study is ongoing to assess efficacy of response to therapy with bevacizumab in the adjuvant setting for surgically resected advanced disease (AVAST-M study).

\section{Antibodies targeting programmed death-1}

Programmed death-1 (PD-1) is an inhibitory cell surface molecule expressed on $\mathrm{T}$ lymphocytes and has homology to CTLA-4. Its ligand PD-L1 is upregulated on tumour cells and thought to be associated with poor prognosis. In addition, senescent/exhausted T lymphocytes (such as those seen within the tumour microenvironment) demonstrate increased expression of PD-1. mABs targeting PD-1 are currently in development for the treatment of melanoma. ${ }^{14}$

\section{Oncolytic vaccine therapy}

Promise has recently been demonstrated with a genetically modified herpes 
simplex virus with selectivity for malignantly transformed cells, in addition to the insertion of a coding sequence for the production of granulocyte-macrophage colony-stimulating factor (GM-CSF) known as OncoVEX ${ }^{\mathrm{GM}-\mathrm{CSF}}$. An overall response rate of $26 \%$ was observed in a phase II study. ${ }^{15}$ A phase III study is now being performed. The agent is injected locally into melanoma metastases and is thought to induce both localised and systemic immune responses with a limited toxicity profile.

\section{Conclusions}

Exciting progress has been made in the treatment of advanced melanoma over the last year. Although overall limited responses have been observed, the responses to treatment with the agents ipilimumab and vemurafenib represent true progress in the treatment of a cancer previously resistant to numerous conventional chemotherapeutic regimens. Knowledge and clinical experience with the application of these agents will most likely pave the way for improved targeted therapies with enhanced efficacy and safety profiles.

\section{Acknowledgements}

The authors acknowledge financial support from the Department of Health via the National Institute for Health Research (NIHR) Comprehensive Biomedical Research Centre Award to Guy's, and St Thomas's NHS Foundation Trust, in partnership with
King's College London and King's College Hospital NHS Foundation Trust.

\section{References}

1 Balch CM, Gershenwald JE, Soong SJ et al. Final version of 2009 AJCC melanoma staging and classification. J Clin Oncol 2009;27:6199-206.

2 Nathan FE, Mastrangelo MJ. Systemic therapy in melanoma. Review. Semin Surg Oncol 1998;14:319-27.

3 Petrella T, Quirt I, Verma S et al; Melanoma Disease Site Group of Cancer Care Ontario's Program in Evidence-based Care. Review. Single-agent interleukin-2 in the treatment of metastatic melanoma: a systematic review. Cancer Treat Rev 2007;33:484-96.

4 Eggermont AM, Suciu S, Santinami M et al. Adjuvant therapy with pegylated interferon alfa- $2 \mathrm{~b}$ versus observation alone in resected stage III melanoma: final results of EORTC 18991, a randomised phase III trial. Lancet 2008;372:117-26.

5 Hodi FS, O'Day SJ, McDermott DF et al. Improved survival with ipilimumab in patients with metastatic melanoma. $N$ Engl J Med 2010;363:711-23.

6 Robert C, Thomas L, Bondarenko I et al. Ipilimumab plus dacarbazine for previously untreated metastatic melanoma. $N$ Engl J Med 2011;364:2517-26.

7 Chapman PB, Hauschild A, Robert C et al. Improved survival with vemurafenib in melanoma with BRAF V600E mutation. $N$ Engl J Med 2011;364:2507-16.

8 Long GV, Kefford RF, Carr PJ et al. Phase $\Omega$ Study of GSK2118436, a Selective Inhibitor of V600 Mutant (Mut)BRAF Kinase: Evidence of Activity in Melanoma Brain Metastases (Mets). Ann Oncol 2010;21:Abstract 12.

9 Infante J, Falchook G, Lawrence D et al. Phase I/II study to assess safety, pharmaco- kinetics and efficacy of the oral MEK1/2 inhibitor GSK1120212 (GSK212) dosed in combination with the oral BRAF inhibitor GSK2118436 (GSK 436). J Clin Oncol 2011;29:CRA8503.

10 Curtin JA, Busam K, Pinkel D, Bastian BC. Somatic activation of KIT in distinct subtypes of melanoma. J Clin Oncol 2006;24:4340-6.

11 Carvajal RD, Antonescu CR, Wolchok JD et al. KIT as a therapeutic target in metastatic melanoma. JAMA 2011;305:2327-34.

12 Nikolaou VA, Stratigos AJ, Flaherty KT, Tsao H. Melanoma: New Insights and New Therapies. J Invest Dermatol 2012 Jan 5.doi:10.1038/jid.2011.421 [Epub ahead of print].

13 Del Vecchio M, Mortarini R, Canova S et al. Bevacizumab plus fotemustine as firstline treatment in metastatic melanoma patients: clinical activity and modulation of angiogenesis and lymphangiogenesis factors. Clin Cancer Res 2010;16:5862-72.

14 Brahmer JR, Drake CG, Wollner I et al. Phase I study of single-agent anti-programmed death-1 (MDX-1106) in refractory solid tumors: safety, clinical activity, pharmacodynamics, and immunologic correlates. J Clin Oncol 2010;28:3167-75.

15 Senzer NN, Kaufman HL, Amatruda T et al. Phase II clinical trial of a granulocytemacrophage colony-stimulating factorencoding, second-generation oncolytic herpesvirus in patients with unresectable metastatic melanoma. J Clin Oncol 2009;27:5763-71.

\section{Address for correspondence: Dr K E} Lacy, St John's Institute of Dermatology, Division of Genetics and Molecular Medicine, King's College London, Guy's Hospital, Guy's Tower Wing, 9th Floor, London SE1 9RT. Email: katie.lacy@kcl.ac.uk 